\title{
Experimental pretesting of message framing to motivate caregiver self-care among parents of children with eating disorders
}

\author{
Autumn Shafer $^{1}$ (i) | Sheetal J. Patel ${ }^{2,3}$ | Cynthia M. Bulik ${ }^{4,5,6}$ | \\ Nancy Zucker ${ }^{7,8}$
}

\author{
${ }^{1}$ School of Journalism and Communication, \\ University of Oregon, Eugene, OR, USA \\ ${ }^{2}$ Student Affairs, Stanford University, Stanford, \\ CA, USA \\ ${ }^{3}$ Passion Dedication Marketing \\ Communications, San Jose, CA, USA \\ ${ }^{4}$ Department of Psychiatry, University of North \\ Carolina, Chapel Hill, NC, USA \\ ${ }^{5}$ Department of Nutrition, University of North \\ Carolina, Chapel Hill, NC, USA \\ ${ }^{6}$ Department of Medical Epidemiology and \\ Biostatistics, Karolinska Institutet, Stockholm, \\ Sweden \\ ${ }^{7}$ Department of Psychiatry and Behavioral Sciences, \\ Duke Center for Eating Disorders, Durham, NC, USA \\ ${ }^{8}$ Department of Psychology and Neuroscience, \\ School of Medicine, Duke University, Durham, \\ NC, USA
}

Correspondence

Autumn Shafer, School of Journalism and Communication, University of Oregon, Eugene, OR, USA.

Email: ashafer@uoregon.edu

Funding information

Carnegie-Knight Foundation, Grant/Award Number: Carnegie-Knight Initiative on the Future of Journalism; University of North Carolina at Chapel Hill, Grant/Award Number:

School of Journalism and Mass Communication
The purpose of this study was to examine the effects of message framing strategies and approach avoidance trait moderators on health communication intervention messages aimed at encouraging adaptive coping among parents of those children with eating disorders (PCEDs). Parents of children with eating disorders $(N=108)$ were randomly assigned in a three-condition (gain frame, loss frame, and control) online message pretesting experiment. Outcome measures included decisional balance (Transtheoretical Model) and behavioral intentions. Gain frames were more beneficial in promoting adaptive behaviors among PCEDs, as loss frames were often harmful. Findings are qualified by approach/avoidance moderator interactions. Formative research benefits from incorporating experimental pretesting methods. Interventions using framed messages should consider relevant personality traits that serve as moderators to behavior adoption.

\section{1 | INTRODUCTION}

Often beginning in adolescence (Engel, Staats Reiss, \& Dombeck, 2007), eating disorders (EDs), including anorexia nervosa, bulimia nervosa, binge ED, and EDs not otherwise specified, affect more than 30 million people in the United 
States (National Eating Disorders Association, 2011). As the course of EDs can be chronic and treatment-resistant, parents of those with an ED may frequently stay highly involved in caregiving for their child well beyond adolescence (Graap et al., 2008).

Research demonstrates that a parent of a child with an eating disorder (PCED) can experience extreme emotional burden when caring for their ill child (Graap et al., 2008; Zucker, Ferriter, Best, \& Brantley, 2005). Concerns over the wellbeing of their child coupled with the demands on a parent's time and energy in meal oversight, treatment costs, and taking care of other family members' needs have been cited as reasons for severe levels of stress. The demands of caregiving seem to exceed the parent's resources and parents experience extreme anxiety related to potential negative outcomes in their child (e.g., Kyriacou, Treasure, \& Schmidt, 2008; Loft, Neumark-Sztainer, \& Croll, 2009). This is a concern in that emotional burden can affect the parent's own health, interfere with a parent's ability to make effective treatment decisions, result in hyper-vigilance, and make the sometimes challenging task of administering treatment even more difficult (Kyriacou et al., 2008; Zucker et al., 2005). Thus, it is important to develop and test strategies that may alleviate some of the stress PCEDs experience.

One such strategy is to encourage the PCEDs to engage in self-care activities as adaptive coping behaviors (Patel, Shafer, Bulik, Brown, \& Zucker, 2014). Adaptive coping behaviors are defined as healthy ways of coping with stress. In this caregiving context, self-care activities are one domain of healthy adaptive behaviors that are selfdirected, separate from one's caregiving responsibilities, and help parents relieve stress and reduce anxiety (Glanz, Rimer, \& Viswanath, 2008). Examples may include engaging in a hobby or taking a walk. When parents do engage in self-care while their child is recovering from an eating disorder, they experience increased energy to follow and administer their child's treatment plan and have more cognitive and physical resources to cope with stress of such management (Zucker et al., 2005).

Even though a parent may understand the negative effects of high levels of stress and anxiety, a key barrier exists that can make a parent reluctant to engage in self-care: the feeling of selfishness for taking time away from a child in crisis (Patel et al., 2014). This barrier can cause parents to feel that they do not deserve or have permission to engage in selfcare or that other people will judge them harshly if they engage in adaptive coping through self-care (Patel et al., 2014).

Eating disorders, especially in the context of parent caregivers as the target audience, have been understudied in health promotion research. This study outlines the quantitative formative research performed to test messages that promoted caregiver adaptive coping that would later appear in eating disorder treatment clinics and online. The theory-driven goal of this study is to test the application of prospect theory and approach/avoidance trait theory to determine how best to communicate with and motivate the PCEDs to engage in coping behaviors that are likely to improve both their own and their child's wellbeing. In addition to the insights for researchers communicating with the PCEDs, the results may be applicable to other health promoters designing messages related to health issues concerned with caregiver coping (e.g., supporting family members of cancer patients). The importance of including experimental pretesting and considering target audience personality traits as part of formative research is also highlighted.

\section{1 | Experimental pretesting as formative research}

Formative message research is conducted during the message design phase of an intervention to assist in the development of effective messages targeted toward a specific audience (Martinez, Latimer, Rivers, \& Salovey, 2012; Noar, 2006; Shafer, Cates, Diehl, \& Hartmann, 2011). Formative research first involves preproduction research, which focuses on better understanding the target audience's attitudes, beliefs, motivations, and behaviors relevant to the intervention health issue (Lindsey et al., 2009; Maddock, Silbanuz, \& Reger-Nash, 2008). Next, production testing involves pretesting developed intervention materials with the specified target audience (Brown, Lindenberger, \& Bryant, 2008; National Cancer Institute, Office of Cancer Communications, 2002). The current study is an example of quantitative production testing, which was done after extensive qualitative preproduction research (Patel et al., 2014).

Without both types of formative research, intervention messages may be counterproductive, causing more harm than good, because they are inappropriate, misunderstood, or not motivating to the target audience (Atkin, 
2001; Fishbein, Hall-Jamieson, Zimmer, von Haeften, \& Nabi, 2002; Martinez et al., 2012; Noar, 2006). Most types of production testing (pretesting) use qualitative methods, such as focus groups or intercept interviews, or perhaps quantitative methods, such as a survey, that ask participants to subjectively report the effectiveness, likability, and attractiveness of the message (Whittingham, Ruiter, Zimbile, \& Kok, 2008). This study advocates for the inclusion of experimental pretesting as a critical component of formative pretesting. That is not to say that qualitative or subjective quantitative methods should not be done, but rather that experimental pretesting should also be performed.

The key benefit of including experimental pretesting in the formative production testing process is that message designers can gather empirical evidence of the message's causal effects on relevant intervention outcomes (Whittingham et al., 2008). If significant effects are found on desired outcome variables, then intervention designers can feel more confident in implementing their intervention. If non-significant or harmful effects are found, then designers can return to earlier stages of their formative research and seek out alternative message designs before time, money, or audience attention are wasted. Another benefit to experimental pretesting is that message designers can compare variations on the message design or content, and through analysis, can determine which variation is most effective at producing the desired outcomes.

\section{2 | Framing adaptive behavior}

According to prospect theory, how a health behavior associated with risk is framed in terms of either a gain or a loss may influence the message's effectiveness (Kahneman \& Tversky, 1979). A gain-framed message highlights the advantages of engaging in a health behavior. A loss-framed message underscores the disadvantages of not performing a health behavior. A number of studies have examined the effectiveness of gain verses loss frames on promoting healthy behaviors (e.g., Cho \& Boster, 2008; Gerend \& Shepherd, 2007; McCall \& Ginis, 2004). One key finding is that the type of health behavior being promoted can be a determining factor in which frame (gain or loss) is more effective (Rothman, Bartels, Wlaschin, \& Salovey, 2006). For prevention behaviors (e.g., brushing teeth to prevent cavities or applying sunscreen to avoid skin cancer), gain frames have been shown to have a small but significant advantage over loss frames at promoting compliance with the health message (O'Keefe \& Jensen, 2007). For detection behaviors (e.g., mammogram to detect breast cancer), research indicates that loss frames are slightly but significantly more effective than gain frames (O'Keefe \& Jensen, 2009). Little is known about the effectiveness of gain or loss frames in promoting adaptive behaviors, which are about managing an illness or stressful event, rather than stopping the illness from happening or recognizing that it is occurring.

To cope with a stressful life event (e.g., child's diagnosis and recovery from an ED), a person may adopt adaptive or maladaptive behaviors (Glanz \& Schwartz, 2008). Adaptive behaviors are healthy ways of coping with stress. Maladaptive behaviors are also ways people cope with stress, but in an unhealthy manner that may ultimately be counterproductive or cause physical or mental harm (e.g., denial, losing sleep, and drinking alcohol).

It would seem that adaptive behaviors share some similarity with prevention behaviors in that they both may be seen as ways to improve health with little risk of harm (Cho \& Boster, 2008; Cohen, 2008; O'Keefe \& Jensen, 2007). Adaptive behaviors, however, are distinct from prevention behaviors because an illness or stress event has already occurred (or may be ongoing), which requires some level of coping. Adaptive behaviors may be seen as a means to return to the healthy state a person was in prior to the stressful event that necessitated the need for coping. The effectiveness of gain and loss frames for prevention and detection behaviors, however, hinge on how risky people think the recommended health behavior is to them. It is possible that PCEDs would perceive self-care as low-risk, if they feel they have nothing to lose and much to gain by engaging in adaptive behaviors. In this instance, it seems gain frames would be more effective at persuading parents to engage in self-care. It is also possible that PCEDs could perceive engaging in self-care to be a high-risk activity if they fear harming their child's recovery by spending time/energy away from their child or if they believe other people will have negative reactions to their self-care. In these circumstances of high-risk perceptions, a loss-framed message might be more 
effective than gain-framed messages at persuading parents to engage in self-care, similar to detection behavior circumstances.

\section{3 | Measuring effectiveness}

The Transtheoretical Model of Behavior Change provides a framework to understand how people move through stages of readiness when in the process of adopting a new behavior (Prochaska, Redding, \& Evers, 2008). The model constructs are helpful in assessing how well a message might move a person through the stages (precontemplation, contemplation, preparation, action, maintenance, and relapse). In this model, as parents move towards engaging in self-care, they would begin to place more importance on the benefits (i.e., feeling less stressed) than the barriers (i.e., feeling selfish). If a message is successful in promoting adaptive behaviors, then participants should place greater importance on the pros and less importance on the cons of participating in self-care. This weighing of the benefits and barriers is called decisional balance and is one way to measure the impact of a message appeal (Prochaska et al., 2008).

\section{4 | Interaction of approach/avoidance tendencies with framing}

As research on framing effects advances, researchers have increasingly sought to investigate moderators of these effects (Covey, 2014). Of the possible moderators of framing, the personality traits associated with the tendency to approach or avoid risk are particularly relevant to self-care for PCEDs, since qualitative research with this population demonstrated that they tended to consider the risks or consequences of their behavior on their child's health outcomes (Patel et al., 2014). These approach/avoidance personality traits are influenced by a person's behavioral approach system (BAS) and behavioral inhibition system (BIS) (Carver \& White, 1994; Shen \& Dillard, 2007). BAS promotes risk seeking, in that people are orientated toward seeking rewards and approaching positive gains. BIS promotes risk aversion by making people weary of potential negative outcomes or harm. A person's approach/avoidance tendencies have been shown to be relatively independent of each other and stable over time and circumstances (Carver \& White, 1994; Shen \& Dillard, 2007; Sutton \& Davidson, 1997).

Previous research shows that levels of BIS and levels of BAS can influence framing effects. There are at least two key reasons to support the assertion that framed messages, which are congruent (match) with an individual's approach and/or avoidance tendencies may be more effective than mismatched messages (Shen \& Dillard, 2007; Sherman, Mann, \& Updegraff, 2006). First, a person may be more likely to notice the benefit or harm information in the gain and loss frames that aligns with their BIS or BAS tendencies. Second, people are likely to have behavioral intentions that are consistent with their BIS or BAS tendencies.

Although more research is needed to build conclusive evidence, a recent meta-analysis found significant effects among several studies that support a congruency effect, such that gain-framed messages should be more effective for people with high BAS tendencies and loss-framed messages should be more effective for people with high BIS tendencies (Covey, 2014). The studies shown to support a significant congruency effect, however, were all tested on prevention behaviors. Adaptive behaviors have not yet been examined in terms of interactions between message frame and BIS or BAS.

Given that there is little empirical evidence of message frame effects on adaptive behaviors or on PCEDs, we propose the following research question:

RQ1: Which frame type (gain or loss) is more effective at persuading PCEDs to engage in self-care?

Given the delicate state of PCEDs, as part of the formative research, with the goal of ensuring that the intervention messages would not do harm to PCEDs, the effects of each frame type were also compared with the PCEDs who saw no message (control condition). The following research question was proposed: 
RQ2: Are gain or loss-framed messages less effective than no message at persuading PCEDs to engage in self-care?

We proposed the following research questions about the presence of a congruency effect:

RQ3: Are gain frames more effective for parents high in approach tendencies (BAS) than loss frames or the control condition?

RQ4: Are loss frames more effective for parents high in avoidance tendencies (BIS) than gain frames or the control condition?

RQ3 and RQ4 provide information about possible interaction effects of the tested messages, which goes beyond the main effects of the frames examined in RQ1 and RQ2.

\section{2 | METHOD}

\section{1 | Participants}

Parents of those children with eating disorders ( $N=108 ; 99 \%$ women, $M_{\text {age }}=52$ years, range: $37-72 ; 99 \%$ Caucasian), were recruited over the course of 1 week by posting a message asking parents to fill out a questionnaire about parent experiences on five ED organization websites for parents, including Families Empowered and Supporting Treatment of Eating Disorders, National Eating Disorders Association, Maudsley Parents, Eating Disorders Coalition, and Around the Dinner Table. The majority of parents were married (81.4\%; 17.6\% divorced/separated; $1.0 \%$ single). Most parents had children with EDs who were female (97.1\%) and the mean age of children with an ED was 20.63 years (range: 9-39). Children had an ED for an average of 6.06 years (range: 1-20). Parents considered themselves highly involved in their child's recovery, $M=4.06$ (scaled from 1-5), $S D=0.96$, and were high seekers of information about EDs, $M=4.53$ (scaled from 1-5), $S D=0.74$.

\section{2 | Procedure and stimulus}

The between-subjects experiment was conducted using Qualtrics online software and was conducted in accordance with Institutional Review Board approval. After completing an informed consent, participants were randomly assigned to read either six gain- or loss-framed statements or to the control condition upon entering the online experiment (gain frame, $n=39$ vs. loss frame, $n=32$ vs. control, $n=37$ ). Participants completed a pre-manipulation survey that assessed BIS/BAS orientation. They were then given as much time as they wanted to read over the six framed statements that were separated into two sets of three (to easily fit on various screen sizes without scrolling). Statement order was randomized to control for any order effects. After each set, participants answered manipulation check measures. Participants in the control condition simply answered the pre and post manipulation questionnaires and were not exposed to any message statements. The content of the gain and loss frames were identical, such that only the frame of the message differed. Gain-framed messages focused on the benefits of engaging in self-care and loss-framed messages focused on the costs of not engaging in self-care (Table 1).

Gain and loss-frame statements were based on the main findings of a previous qualitative study with PCEDs and ED treatment experts who have frequent contact with the parents of their patients, which concluded that key elements to engaging in self-care activities were helping the child recover and reducing emotional burden (Patel et al., 2014). After exposure to the statements, participants answered the study questionnaire, including the dependent measures and demographic information. Participants were debriefed and compensated $\$ 5$ for their time. 
TABLE 1 Statements about engaging in self-care by message frame

Gain-framed messages

Experts in eating disorders and parents going through the same things as you agree that:

Even just getting enough sleep will allow you to set a good example as a role model for healthy habits that could positively impact your family's health, especially that of your child

Taking part in basic self-care, such as a 5-min walk, will enhance your ability to care for your child

Ultimately, if you take time to care for yourself, you will help your child recover

Taking time to care for yourself, will allow you to feel liberated and close to the people in your life

If you talk about your child's eating disorder with other parents, you will be supported by people who really understand what you are going through

Taking $10 \mathrm{~min}$ to relax and enjoy a hobby will allow you to be less anxious throughout the day
Loss-framed messages

Even just getting a lack of sleep will make you a role model for unhealthy habits that could negatively impact your family's health, especially your child

Not taking part in basic self-care, such as a 5-min walk, will increasingly diminish your ability to care for your child

Ultimately, if you don't take time to care for yourself, you may get in the way of your child's recovery

Failure to take time to care for yourself will make you feel more and more trapped and isolated from other people in your life

If you don't talk about your child's eating disorder with other parents, you will increasingly feel that people don't understand what you are going through

Not taking 10 min to relax and enjoy a hobby will make you increasingly more anxious throughout the day

\section{3 | Measures}

\subsection{1 | Manipulation check}

On the cognitive dimension, the effectiveness of the frame treatment manipulation was assessed with an index consisting of four Likert-type items ( 1 = strongly disagree, 5 = strongly agree). Participants were asked to rate how much they agreed with statements about the messages they had just viewed (e.g., "It is mostly about the bad things that could happen if you don't regularly engage in self-care activities," and "It highlights the positive aspects of engaging in self-care activities"). Items were reverse-coded when necessary with a higher score indicating agreement that gain frames were present (Cronbach's $\alpha=.88$ ). These items were adapted from Cho and Boster (2008). Another index of four semantic differentials measures (e.g., "Happy/Sad," "Positive, Negative") anchored on a 7-point scale and adapted from previous studies asked participants to rate how the messages made them feel and were reversecoded when necessary, with higher scores associated with gains and lower scores with losses (Cronbach's $\alpha=.88$ ) (e.g., Cho \& Boster, 2008).

\subsection{2 | Behavioral inhibition (BIS) and approach (BAS) systems}

Behavioral inhibition system was measured using seven Likert-type items ( $1=$ strongly disagree, $5=$ strongly agree), which asked participants to rate their agreements with certain statements (e.g., "I feel pretty worried or upset when I think somebody is angry at me"). BAS was measured using 13 Likert-type items ( 1 = strongly disagree, 5 = strongly agree), which asked participants to rate their agreement with certain statements (e.g., "I often act on the spur of the moment"). These items were taken directly from Carver and White (1994). Cronbach's $\alpha$ was .81 for BIS (M $=3.73$, $S D=0.60)$ and .85 for BAS $(M=3.21, S D=0.51)$.

\subsection{3 | Decisional balance}

Decisional balance was measured by asking participants to rate how important the key pro ("Feeling less stressed") and the key con ("Feeling selfish") were ( 1 = not at all important, 5 = extremely important) to willingness to engage in self-care 
activities. The content of these items were based on extensive qualitative research with PCEDs about the key benefits and barriers they perceived related to engaging in self-care (Patel et al., 2014) and was also highlighted in the message stimuli. The format for these items was adapted from another Transtheoretical Model scale (Cancer Prevention Research Center, 1991). Reliability is not provided, as these were single item measures that were examined separately.

\subsection{4 | Behavioral intentions}

Behavioral intentions to engage in self-care activities were measured using two items, which asked participants how likely they would be ( 1 = very unlikely, 5 = very likely) to engage in specific self-care activities (i.e., "I will take personal time for myself," "I will participate in a personal hobby or activity I enjoy"). These items were developed based on qualitative data (Patel et al., 2014) and also highlighted in the message stimuli. The question format was adapted from another study that used the Transtheoretical Model (Cancer Prevention Research Center, 1991). Reliability is not provided as each item represented a distinct type of behavior and was examined separately.

\section{4 | Analysis}

To examine the research questions of which frame type (gain or loss) was more effective at persuading PCEDs to engage in self-care (RQ1), whether either frame type (gain or loss) was less effective than no message (RQ2), and if there were any congruency interaction effects, such that gain frames would be more effective for participants high in BAS (RQ3) and loss frames would be more effective for participants high in BIS (RQ4), a series of multiple regression analyses with interactions was conducted. The decisional balance items and each behavioral intention were in turn predicted from (a) experimental framing condition (gain vs. loss vs. control), (b) the potential moderating variables of motivational orientation (BIS and BAS), (c) the two-way interaction terms, and (d) the three-way interaction terms. Results were interpreted in the highest model for which there was a significant R-squared change present (West, Aiken, \& Krull, 1996). Since the categorical independent variable had three levels, variables were dummy coded and the R-Squared change test (from model summary) in the step the interaction term was added was the overall test of the model significance. When a significant interaction emerged, it was decomposed using a simple slopes analysis at $1 S D$ above and below the mean $(-1 S D=$ low and $+1 S D=$ high) (Aiken \& West, 1991). None of the three-way interactions were predicted or significant in any of the analyses, and thus, will not be discussed further.

\section{3 | RESULTS}

\section{1 | Manipulation check}

\subsection{1 | Cognitive dimension}

A $t$ test indicated the framing manipulations produced significant differences in perceptions of gains or losses by condition, $t(66)=-8.20, p<.001$. Parents who were in the gain-framed message condition thought the messages they viewed promoted the gains of self-care and not the losses of not engaging $(M=3.99, S D=0.64)$, whereas those in the loss-frame condition thought the messages they saw promoted the losses associated with not engaging in self-care, but not the gains if self-care was taken $(M=2.67, S D=0.70)$. Thus, the manipulations were successful for this dimension.

\subsection{2 | Affective dimension}

A $t$-test indicated that gain- and loss-frame conditions successfully induced differential amounts of positive and negative emotions. The gain-framed condition generated a greater amount of positive emotion $(M=4.58, S D=0.94)$ than the loss-frame condition ( $M=3.58, S D=1.30), t(59)=-3.45, p<.005$. 
TAB LE 2 Framed condition $\times$ BIS orientation regression model for feeling less stressed (gain as reference group)

\begin{tabular}{|c|c|c|c|c|}
\hline & $B$ & SE & $\beta$ & $t$ \\
\hline Intercept & 4.414 & .125 & & $35.320^{* * *}$ \\
\hline BIS & -0.599 & .233 & -.472 & -2.575 \\
\hline BAS & 0.433 & .328 & .289 & 1.323 \\
\hline Loss & -0.395 & .179 & -.243 & $-2.207^{*}$ \\
\hline Control & -0.059 & .183 & -.037 & -0.322 \\
\hline $\mathrm{BIS} \times \mathrm{BAS}$ & -0.182 & .190 & -.124 & -0.955 \\
\hline Con. $\times$ BAS & -0.193 & .447 & -.072 & -0.432 \\
\hline Loss $\times$ BAS & 0.159 & .412 & .072 & 0.385 \\
\hline Con. $\times$ BIS & 0.881 & .326 & .407 & $2.701^{* *}$ \\
\hline Loss $\times$ BIS & 0.324 & .312 & .155 & 1.038 \\
\hline
\end{tabular}

BIS, behavioral inhibition system; BAS, behavioral approach system.

$R^{2}=.22$

${ }^{*} p<.05,{ }^{* *} p<.01,{ }^{* * *} p<.001$.

\subsection{3 | Behavioral inhibition system/Behavioral approach system}

It was important to test that BIS/BAS tendencies were randomly distributed in each condition and as expected, no significant differences in BIS or BAS orientation by experimental condition were found (all $p>.14$ ).

\section{2 | RQ1: Gain vs. loss frame main effects}

Results were interpreted at the two-way interaction model level for the pro of importance of feeling less stressed as motivation to engage in self-care, which was significant, F-change $(2,91)=3.67, p<.05$, $R^{2}$-change $=.06$. Gain frames were significantly more effective than loss frames at increasing participants' importance of feeling less stressed as a motivation to engage in self-care $(b=-0.40, S E=.18), t(91)=-2.21$, $p<.05$ (see Table 2).

No significant results for the con of feeling selfish as a deterrent to engaging in self-care, intention to take personal time (as self-care), or intention to engage in a hobby (as self-care) were found between the gain and loss conditions.

\section{3 | RQ2: Gain and loss frames vs. no message}

Results were interpreted at the two-way interaction model level for intention to take personal time (as self-care), which was significant, $F$-change $(2,91)=4.33, p<.05, R^{2}$-change $=.07 . R Q 2$ asked if there were any harmful main effects of frame type, such that gain or loss-framed messages were worse than no message (control condition). RQ2 was answered through a main effect for loss frames compared with the control $(b=-0.63, S E=.31), t(91)=-2.01$, $p<.05$, such that, participants who saw the loss-framed messages reported lower intentions to take personal time compared with participants who did not see any message (see Table 3). No harmful main effects were found for the gain-framed messages.

Neither frame type was significantly different from the control with regard to the importance of feeling less stressed as a motivator (see Table 4), the con of feeling selfish as a deterrent to engaging in self-care, or intentions to engage in a hobby (as self-care) (See Table 5). 
TABLE 3 Framed condition $\times$ BIS orientation regression model intention to take personal time (control as reference group)

\begin{tabular}{|lllcc|} 
& $\boldsymbol{B}$ & $\boldsymbol{S E}$ & $\boldsymbol{\beta}$ & $\boldsymbol{t}$ \\
\hline Intercept & 4.178 & .221 & & $18.929^{* * *}$ \\
\hline BIS & -0.076 & .360 & -.036 & -0.211 \\
\hline BAS & 0.012 & .456 & .005 & 0.025 \\
\hline Loss & -0.630 & .314 & -.233 & $-2.005^{*}$ \\
\hline Gain & -0.188 & .303 & -.071 & -0.619 \\
\hline BIS $\times$ BAS & -0.067 & .315 & -.027 & -0.212 \\
\hline Gain $\times$ BAS & 0.747 & .712 & .134 & 1.049 \\
\hline Loss $\times$ BAS & 0.925 & .630 & .252 & 1.469 \\
\hline Gain $\times$ BIS & -1.209 & .539 & -.303 & $-2.241^{*}$ \\
\hline Loss $\times$ BIS & 0.190 & .555 & .055 & 0.342 \\
\hline
\end{tabular}

BIS, behavioral inhibition system; BAS, behavioral approach system.

$R^{2}=.23$.

${ }^{*} p<.05,{ }^{* * *} p<.001$.

TAB LE 4 Framed condition $\times$ BIS orientation regression model for feeling less stressed (control as reference group)

\begin{tabular}{lllcc|} 
& $\boldsymbol{B}$ & $\boldsymbol{S E}$ & $\boldsymbol{\beta}$ & $\boldsymbol{t}$ \\
\hline Intercept & 4.355 & .133 & & $32.648^{* * *}$ \\
\hline BIS & 0.281 & .218 & .222 & 1.293 \\
\hline BAS & 0.073 & .276 & .049 & 0.264 \\
\hline Loss & -0.336 & .190 & -.207 & -1.770 \\
\hline Gain & 0.059 & .183 & .037 & 0.322 \\
\hline BIS $\times$ BAS & -0.182 & .190 & -.124 & -0.955 \\
\hline Gain $\times$ BAS & 0.361 & .430 & .108 & 0.838 \\
\hline Loss $\times$ BAS & 0.488 & .381 & .222 & 1.282 \\
\hline Gain $\times$ BIS & -0.881 & .326 & -.368 & $-2.701^{*}$ \\
\hline Loss $\times$ BIS & -0.557 & .335 & -.267 & -1.659 \\
\hline
\end{tabular}

BIS, behavioral inhibition system; BAS, behavioral approach system.

$R^{2}=.22$.

${ }^{*} p<.05,{ }^{* * *} p<.001$.

\subsection{RQ3 and RQ4: congruency effects}

To answer RQ3, if gain frames were most effective for individuals high in BAS, decomposition analyses were conducted for condition differences at three levels of BAS (low, mean, and high), no significant differences by frame type at any level of BAS emerged for any of the outcome variables.

To answer RQ4, if loss frames were most effective for individuals high in BIS, decomposition analyses were conducted for condition differences at three levels of BIS (low, mean, and high). Individuals high in avoidance motivation reported that feeling less stressed was less important after exposure to the loss-framed message than exposure to the control condition ( $b=0.67, \mathrm{SE}=.24), t(91)=2.76, p<.01$ ( $n$ s between gain and control or gain and loss at high BIS) (see Figure 1). Thus, contrary to congruency expectations, loss frames were less effective than no message, as they decreased the importance of this pro for individuals high in avoidance. No other congruency effects for RQ4 were found among the remaining outcome variables. 
TAB LE 5 Framed condition $\times$ BIS orientation regression model for intention to engage in a hobby or activity (control as reference group)

\begin{tabular}{|c|c|c|c|c|}
\hline & $B$ & SE & $\boldsymbol{\beta}$ & $t$ \\
\hline Intercept & 4.139 & .226 & & $18.335^{* * *}$ \\
\hline BIS & 0.073 & .368 & .035 & 0.198 \\
\hline BAS & 0.467 & .139 & .742 & 0.460 \\
\hline Loss & -0.499 & .321 & -.184 & -1.555 \\
\hline Gain & -0.175 & .310 & -.066 & -0.563 \\
\hline $\mathrm{BIS} \times \mathrm{BAS}$ & -0.208 & .322 & -.085 & -0.647 \\
\hline Gain × BAS & 0.431 & .728 & .077 & 0.592 \\
\hline Loss $\times$ BAS & 0.115 & .644 & .031 & 0.178 \\
\hline Gain × BIS & -1.448 & .552 & -.363 & $-2.624^{*}$ \\
\hline Loss $\times$ BIS & 0.048 & .568 & .014 & 0.085 \\
\hline
\end{tabular}

BIS, behavioral inhibition system; BAS, behavioral approach system.

$R^{2}=.19$.

${ }^{*} p<.05,{ }^{* * *} p<.001$.

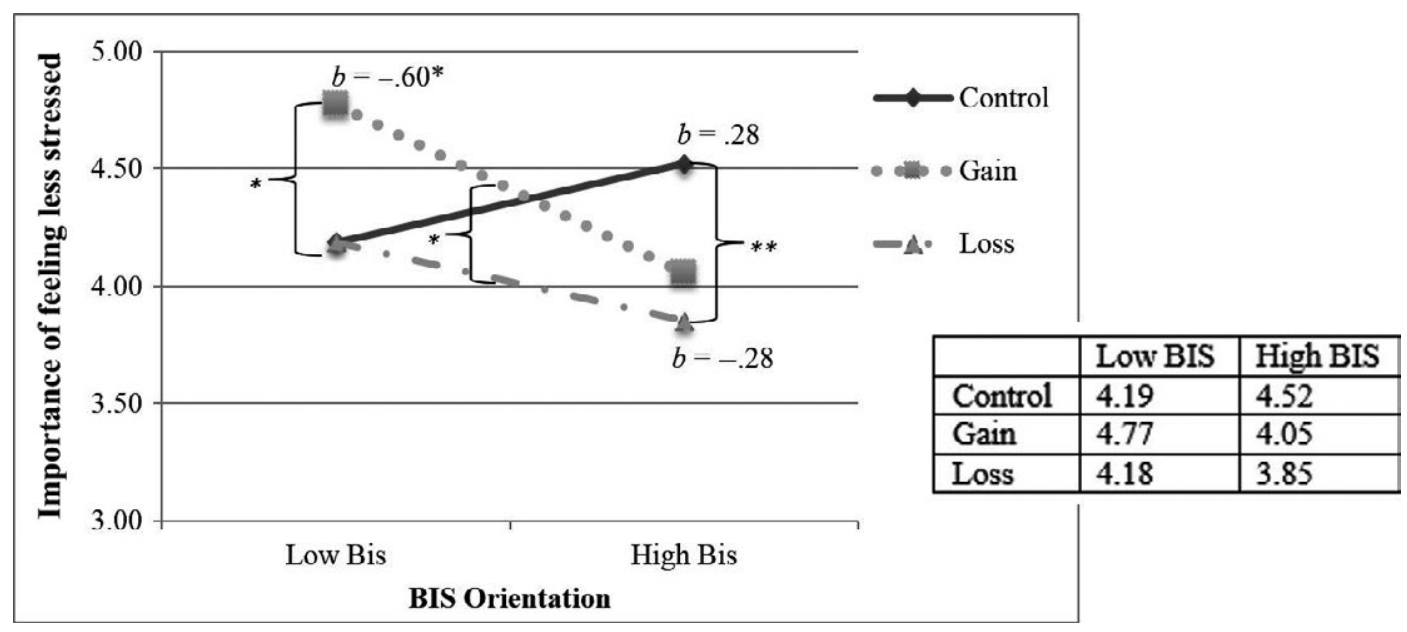

FIGURE 1 Importance of feeling less stressed as a function of exposure to framed message or control conditions and behavioral inhibition system (BIS) orientation

Note: "How important are each of the following as influences on your willingness to engage in self-care activities?: Feeling Less stressed." Results plotted at \pm 1 SD of BIS. ${ }^{*} p<.05,{ }^{* *} .01,{ }^{* * *} p<.001$.

\section{5 | Decomposition analyses}

\subsection{1 | Pro: feeling less stressed as a motivation to engage in self-care}

Although not originally part of our research questions, decomposition analyses on the interaction effect of BIS and condition revealed more information about frame type effectiveness, which was useful as formative research. For individuals low in avoidance motivation, feeling less stressed was more important to participants exposed to the gain-framed message than to loss-framed message $(b=0.59, S E=.26), t(91)=2.36, p<.05$, or the control condition $(b=0.58, S E=.28), t(91)=2.09, p<.05$ (ns between loss and control conditions at low BIS). At the mean of BIS, individuals reported feeling less stressed was more important after exposure to the gain-framed message than to 
exposure to the loss-framed message $(b=0.39, S E=.18), t(91)=2.21, p<.05$ (ns between gain and control or loss and control at mean BIS). This means that gain frames were successful at increasing the importance of feeling less stressed when thinking about engaging in self-care for low and mean avoidance individuals. Simple slopes analyses revealed that as BIS tendencies increased, the effectiveness of a gain-framed message decreased $(b=-0.60, p<.05)$ (see Figure 1).

\subsubsection{Intentions to take person time (as self-care)}

Decomposition analyses on the interaction effect of BIS and condition revealed information that was beyond our original research questions, yet included because of its value as formative research. Decomposition analyses indicated that for participants low in avoidance orientation, exposure to the gain-framed message led to greater intentions to take personal time compared with the loss condition $(b=1.28, S E=.43$ ), $t(91)=2.97, p<.005$ (no significant difference between gain or control or loss and control at low BIS existed). Among individuals high in avoidance motivation, reading the gain-framed messages resulted in less intention to take personal time than the control condition $(b=-0.91, S E=.42), t(91)=-2.17, p<.05$ (no significant difference between gain or loss or loss and control at high BIS existed). Simple slopes analyses found that as BIS tendencies increased, the effectiveness of gain-framed messages decreased $(b=-0.91, p<.05)$ (see Figure 2 ).

\subsubsection{Intention to engage in a hobby (as self-care)}

These decomposition analyses did reveal that for participants low in avoidance orientation, exposure to the gainframed message led to greater intentions to engage in a hobby compared with the loss condition $(b=1.22, S E=.44)$, $t(91)=2.77, p<.01$ (no significant difference between gain or control or loss and control at low BIS existed). Among individuals high in avoidance motivation, reading the gain-framed messages resulted in less intention to engage in a hobby than the control condition ( $b=-1.042$, SE $=.43$ ), $t(91)=-2.425, p<.05$ (no significant difference between gain or loss or loss and control at high BIS existed). Similar to findings from other dependent variables a simple slopes analyses found that gain frames were more effective at influencing parents low in avoidance traits than those high in avoidance traits $(b=-1.37, p<.001)$ (see Figure 3 ).

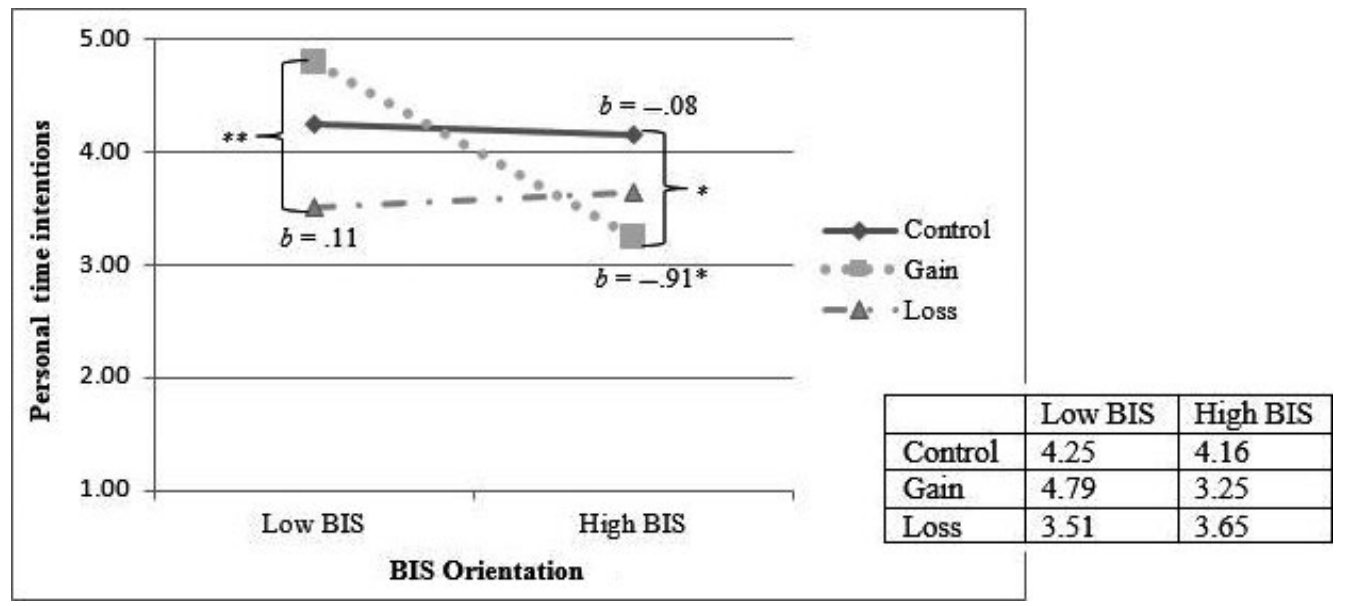

FIGURE 2 Intentions to take personal time as a function of exposure to framed message or control conditions and behavioral inhibition system (BIS) orientation Note: "I will take personal time for myself." Results plotted at \pm 1 SD of BIS. ${ }^{*} p<.05,{ }^{* *} .01,{ }^{* * *} p<.001$. 


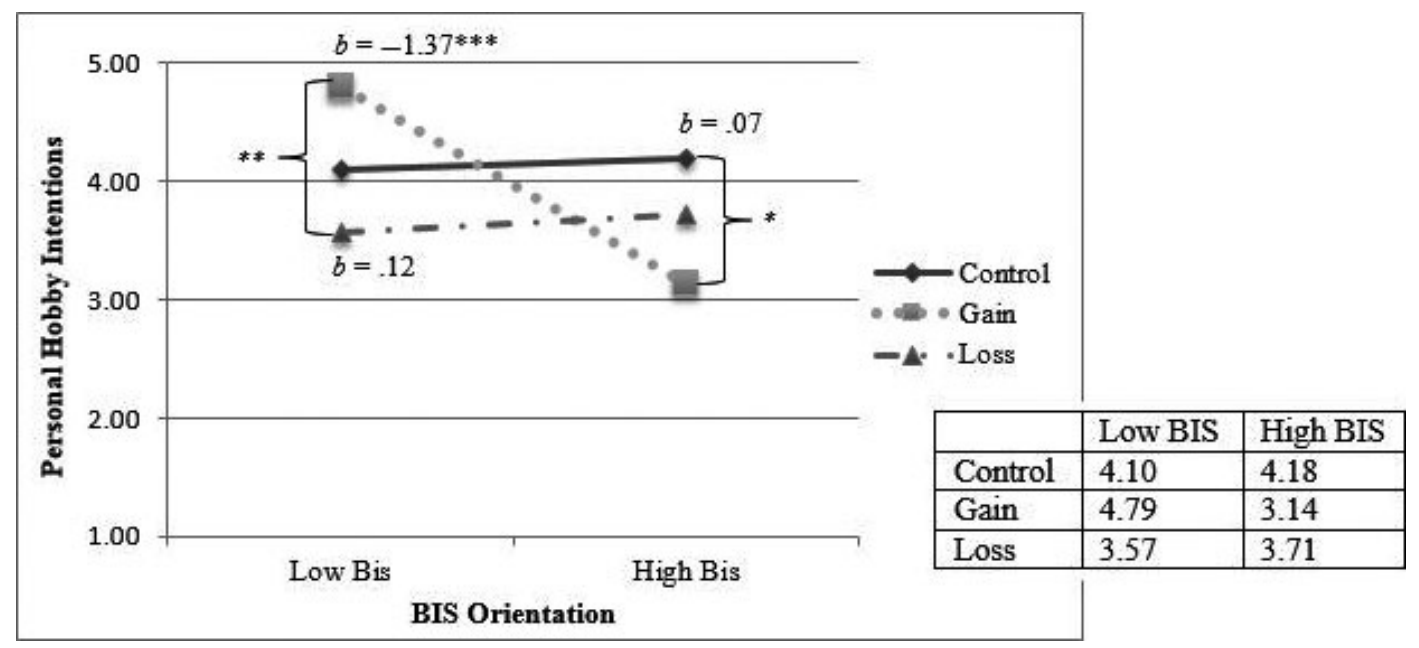

FIGURE 3 Intentions to engage in a personal hobby or activity as a function of exposure to framed message or control conditions and behavioral inhibition system (BIS) orientation

Note: "I will participate in a personal hobby or activity I enjoy." Results plotted at \pm 1 SD of BIS. ${ }^{*} p<.05,{ }^{* *} .01,{ }^{* * *} p<.001$.

\section{4 | DISCUSSION}

The present study is one of few to examine health messages that promote adaptive behaviors, in this case, for PCEDs and to investigate message-framing effects on self-care decisional balance and intentions. The results suggest that it is evident that avoidance orientation plays a role in moderating gain framing effects. Research in this area that only looks at exposure to gain and loss frames without considering the moderating effects of avoidance orientation is unlikely to fully explain the framing effects.

\subsection{RQ1: Gain vs. loss frame main effects}

With regard to RQ1 (which frame might be more effective at increasing adaptive behaviors for PCEDs) the pattern of results indicate an advantage towards gain frames, which increased the importance of feeling less stressed as a motivation to engage in self-care compared with loss frames. Although, no other main effects for gain versus loss emerged, moderated effects discussed later appear to support gain over loss frames for this study, such that increased intentions to engage in adaptive behaviors existed, such as taking personal time and involving oneself in a hobby, for those parents who have lower avoidance tendencies.

\subsection{RQ2: Gain and loss frames vs. no message}

In terms of RQ2 (which asked about harmful main effects of frame type compared with receiving no message), there was a main effect for loss frames, such that respondents exposed to the loss frames were less likely to intend to take personal time than control group participants. It is possible that loss frames remind parents about associated risk with conducting this particular adaptive behavior and include too much negative information for parents who are already stressed and anxious. In this case, loss frames may need to be avoided when creating messages for PCEDs. Without experimental pretesting of these messages, finding the harmful effects of frames may not have been possible, making this type of formative research valuable. 


\section{3 | RQ3 and RQ4: congruency effects}

RQ3 and RQ4 examined congruency (also called matching) effects asking if there was a congruency effect between gain frames and BAS, such that gain frames would be most effective with participants high in BAS (RQ3) and if a congruency effect existed between loss frames and BIS, such that loss frames would be most effective with participants high in BIS (RQ4). No matching effects for high BIS/loss or high BAS/gain were found.

One possible explanation for this absence of a matching effect is that given the amount of emotional burden and anxiety already felt by PCEDs, loss messages invoked too much fear to be effective, and actually became harmful, and gain-framed messages do not remove enough negative emotion to be effective. This fear explanation should be explored in future research.

Taken from another perspective, matching effects predict that a certain type of frame will be most effective at high levels of BIS or BAS, which implies that we might expect opposite directional relationships between gain/BAS and loss/BIS at low levels of approach or avoidance. Thus, it might also be important to consider how message frame and approach/avoidance interact across the continuum of BIS and BAS rather than looking for a matching effect only at high levels of BIS or BAS, as indicated by study findings. This study found no matching effects across the continuum of BAS for gain fames. Yet, the degree of avoidance motivation was important when BIS tendencies were low, loss frames were significantly less effective than gain frames (e.g., feeling less stressed, intentions to take personal time, and intentions to engage in a hobby), supporting the congruency effect hypothesis for loss frames and BIS.

At the same time, significant results were found for gain and BIS, such that gain frames were most effective at low BIS levels and least effective at high BIS levels for the pro of importance of feeling less stressed and the intention to take personal time or engage in a hobby. In terms of the congruency effect, for risk aversion, the study results suggest that people with low avoidance orientation react in similar fashion to how theory suggests those high in approach orientation react, in that they may respond better to positive benefit frames. Given that only partial support for the congruency effect was found, this also suggests that the congruency effect should be further studied in terms of adaptive behaviors.

Lastly, neither gain- nor loss-frame messages were effective at making the pro of engaging in self-care more important, con of engaging in self-care less important, or increasing behavioral intentions for those parents who have higher risk avoidance tendencies. In fact, either frame can be more harmful than seeing no message at all for people who have high BIS levels (high in risk avoidance). Therefore, both frames (especially loss frames) may not be as effective for those high in risk avoidance traits.

When examining how to frame messages, this quantitative, experimental, formative research shows that adaptive behaviors are different from prevention and detection behaviors, in that a person is already experiencing a health risk rather than considering how to prevent it or what it would be like if they were diagnosed (detection). In this case, PCEDs are already in crisis and potentially risk sensitive, so the stakes of a risk may seem higher. Therefore, when creating messages for PCEDs, the results suggest that gain frames may be effective for everyone except those with high avoidance orientation (high levels of BIS). For these parents, it may be necessary to increase exposure to messages in a longer campaign, which may help reiterate the positive benefits of engaging in self-care. Additionally, tailored messaging as opposed to mass or targeted communication may be needed. This could be accomplished through one-on-one communication with treatment team members, or other media vehicles that allow for tailoring, such as a text-messaging campaign or direct mail (see Hawkins, Kreuter, Resnicow, Fishbein, \& Dijkstra, 2008). The results also indicate that when developing messages for encouraging adaptive behaviors, it is important to conduct formative research to determine if risk avoidance traits may influence message effectiveness.

\section{4 | Practical communication implications}

In addition to theoretical implications, the results have important practical implications for development of mass communication and patient-provider dialog promoting adaptive behaviors for PCEDs. Research suggests that parents 
experience high emotional burden and stress when caring for a child with an ED. It is important that parents engage in healthy adaptive behaviors to help their children recover. Our findings reveal that the use of loss frames are more likely to be harmful rather than helpful in encouraging parents to engage in self-care. Therefore, it may be better for healthcare professionals and health communication practitioners to use more positively framed messages, linking adaptive behavior for caregivers to the ability to feel less stressed and to better aid in the recovery of a child with an ED.

This same method may apply for other caregivers and should be examined in future research, such as parents caring for a child ill with cancer or another serious medical condition or adults who are taking care of their aging parents, where emotional burden and stress may be high, and caregivers may face similar barriers to engaging in selfcare. It may also be beneficial to examine perceived risk between these different populations to find the differences in framing and approach/avoidance effects.

\section{5 | Limitations}

Limitations of the present study provide further opportunities for future research. The online collection of data may be a concern because of the less control of nuisance variables, as the study did not take place in a physical lab under researcher observation; although, random assignment should have spread potential nuisance variables among the conditions. Descriptive analyses revealed that participants spent approximately the same amount of time per condition ( $M=24.27 \mathrm{~min}, S D=9.90$ ), indicating that the experiment was completed in one sitting by most respondents, thus lessening the threat that external events could have influenced respondents' answers between exposure to the stimulus and post-test. Online data collection was deemed necessary for this study because of the difficulty in finding PCEDs willing to speak because of the stigma and societal blame associated with being a parent of child with an ED (Loft et al., 2009; Patel et al., 2014). Children with EDs can be secretive and impose boundaries on parents speaking about their ED. Therefore, conducting this experiment online allowed parents to participate without the burden of potentially facing some of these barriers. A related limitation was the sample size. A post hoc power analysis was conducted using GPower (Faul, Erdfelder, Buchner, \& Lang, 2009) for the sample size of 108 with nine predictor variables and $\alpha$ level of $p<.05$. The post hoc analysis revealed that the statistical power for this study was .12 for a small effect $\left(f_{2}=.02\right), .78$ for a medium effect $\left(f_{2}=.15\right)$, and .99 for detecting a large effect $\left(f_{2}=.35\right)$ (Cohen, 1988). The sample size was, therefore, likely sufficient to detect a medium or large effect size, but far below adequate power (i.e., .80) for detection of a small effect.

A limitation may be the use of single item measures as dependent variables, however, based on our previous qualitative work, the issues represented by each item were the main issues relevant to this population, which we sought to test with this formative research. Next, the sample of parents was very similar demographically. Previous research has suggested that parents of other ethnicities and economic statuses and parents just learning of their child's diagnosis may respond differently, given differences in perceived risk of engaging in self-care. Lastly, we only examine adaptive behaviors for PCEDs, and as suggested, further research could determine if there are similar effects for different populations and health issues. Future research might also consider whether known moderators of framing effects, such as self-efficacy and regulatory focus, also apply within the context of adaptive behaviors.

\section{5 | CONCLUSIONS}

The findings in this study provide evidence that gain frames may be more beneficial in promoting healthy adaptive behaviors among PCEDs, while loss frames may be harmful. These finding are qualified by approach/avoidance tendencies, such that those high in avoidance, do not seem to benefit from either frame while those low in avoidance and those low or high in approach individuals may receive benefits from messaging. The study also shows that there is a need for further research to examine the theoretical relationships between message framing, individual 
difference moderators, and promotion of healthy adaptive behaviors, as adaptive behaviors are imperative for many people coping with health conditions.

\section{FUNDING AND ACKNOWLEDGMENTS}

The authors thank the Carnegie-Knight Foundation for the Carnegie-Knight Initiative on the Future of Journalism Grant, the University of North Carolina at Chapel Hill for the School of Journalism and Mass Communication developmental grant, and Professor Jane Brown, all of whom made this study possible

\section{CONFLICT OF INTEREST}

The authors have no conflicts of interest to disclose.

\section{REFERENCES}

Aiken, L., \& West, S. (1991). Multiple regression: Testing and interpreting interactions. Thousand Oaks, CA: Sage.

Atkin, C. K. (2001). Theory and principles of media health campaigns. In R. E. Rice, \& C. K. Atkin (Eds.), Public communication campaigns (pp. 49-68). Thousand Oaks, CA: Sage.

Brown, M. K., Lindenberger, J. H., \& Bryant, C. A. (2008). Using pretesting to ensure messages and materials are on strategy. Health Promotion Practice, 8(2), 116-122.

Cancer Prevention Research Center (1991). Transtheoretical model measures. Retrieved from http://web.uri.edu/cprc/ measures/

Carver, C., \& White, T. (1994). BIS/BAS scales. Journal of Personality and Social Psychology, 67(2), 319-333.

Cho, H., \& Boster, F. (2008). Effects of gain versus loss frame antidrug ads on adolescents. Journal of Communication, 58(3), 428-446.

Cohen, J. (1988). Statistical power analysis for the behavioral sciences (2nd ed.). Hillsdale, NJ: Lawrence Erlbaum.

Cohen, E. (2008). 'My loss is your gain': Examining the role of message frame, perceived risk, and ambivalence in the decision to become an organ donor. Conference Papers-International Communication Association, Montreal, QC, 1-39.

Covey, J. (2014). The role of dispositional factors in moderating message framing effects. Health Psychology, 33(1), 52-65.

Engel, B., Staats Reiss, N., \& Dombeck, M. (2007). Prevalence, onset and course of eating disorders. Retrieved from www.mentalhelp.net/poc/view_doc.php?type=doc\&id=11746\&cn=46

Faul, F., Erdfelder, E., Buchner, A., \& Lang, A.-G. (2009). Statistical power analyses using G*Power 3.1: Tests for correlation and regression analyses. Behavior Research Methods, 41, 1149-1160.

Fishbein, M., Hall-Jamieson, K., Zimmer, E., von Haeften, I., \& Nabi, R. (2002). Avoiding the boomerang: Testing the relative effectiveness of antidrug public service announcements before a national campaign. American Journal of Public Health, 92(2), 238-245.

Gerend, M., \& Shepherd, J. (2007). Using message framing to promote acceptance of the human papillomavirus vaccine. Health Psychology, 26(6), 745-752.

Glanz, K., Rimer, B. K., \& Viswanath, K. (2008). Health behavior and health education: Theory, research, and practice (3rd ed.). San Francisco, CA: Jossey-Bass.

Glanz, K., \& Schwartz, M. D. (2008). Stress, coping, and health behavior. In K. Glanz, B. K. Rimer, \& K. Viswanath (Eds.), Health behavior and health education: Theory, research, and practice (4th ed., pp. 211-230). San Francisco, CA: Jossey-Bass.

Graap, H., Bleich, S., Herbst, F., Trostmann, Y., Wancata, J., \& de Zwaan, M. (2008). The needs of carers of patients with anorexia and bulimia nervosa. European Eating Disorders Review: The Journal of the Eating Disorders Association, 16(1), 21-29.

Hawkins, R. P., Kreuter, M., Resnicow, K., Fishbein, M., \& Dijkstra, A. (2008). Understanding tailoring in communicating about health. Health Education Research, 23(3), 454-466.

Kahneman, D., \& Tversky, A. (1979). Prospect theory: An analysis of decision under risk. Econometrica, 47(2), $263-291$.

Kyriacou, O., Treasure, J., \& Schmidt, U. (2008). Understanding how parents cope with living with someone with anorexia nervosa: Modeling the factors that are associated with caregiver distress. International Journal of Eating Disorders, 41(3), 233-242.

Lindsey, L. M., Silk, K. J., Von Friederichs-Fitzwater, M. M., Hamner, H. C., Prue, C. E., \& Boster, F. J. (2009). Developing effective campaign messages to prevent neural tube defects: A qualitative assessment of women's reactions to advertising concepts. Journal of Health Communication, 14(2), 131-159. 
Loft, K. A., Neumark-Sztainer, D., \& Croll, J. K. (2009). Informing family approaches to eating disorder prevention: Perspectives of those who have been there. International Journal of Eating Disorders, 42(2), 146-152.

Maddock, J. E., Silbanuz, A., \& Reger-Nash, W. (2008). Formative research to develop a mass media campaign to increase physical activity and nutrition in a multiethnic state. Journal of Health Communication, 13, 208-215.

Martinez, J. L., Latimer, A. E., Rivers, S. E., \& Salovey, P. (2012). Formative research for a community-based message-framing intervention. American Journal of Health Behavior, 36(3), 335-347.

McCall, L. A., \& Ginis, K. A. M. (2004). The effects of message framing on exercise adherence and health beliefs among patients in a cardiac rehabilitation program. Journal of Applied Biobehavioral Research, 9(2), 122-135.

National Cancer Institute, Office of Cancer Communications (2002). Making health communication programs work: A planner's guide. NIH Pub. No. 02-5145. U.S.: Department of Health \& Human Services, National Institutes of Health, National Cancer Institute, Baltimore, MD.

National Eating Disorders Association (2011) Get the facts on eating disorders. Retrieved from http://www.nationaleatingdis orders.org/get-facts-eating-disorders

Noar, S. M. (2006). A 10-year retrospective of research in health mass media campaigns: Where do we go from here? Journal of Health Communication, 11(1), 21-42.

O'Keefe, D., \& Jensen, J. (2007). The relative persuasiveness of gain-framed and loss-framed messages for encouraging disease prevention behaviors: A meta-analytic review. Journal of Health Communication, 12(7), 623-644.

O'Keefe, D., \& Jensen, J. (2009). The relative persuasiveness of gain-framed and loss-framed messages for encouraging disease detection behaviors: A meta-analytic review. Journal of Communication, 59(2), 296-316.

Patel, S., Shafer, A., Bulik, C. M., Brown, J. D., \& Zucker, N. L. (2014). Parents of children with eating disorders: Developing theory-based health communication messages to promote caregiver wellbeing. Journal of Health Communication, 19, 593-608.

Prochaska, J., Redding, C., \& Evers, K. (2008). The transtheoretical model and stages of change. In R. Glanz, B. Rimer \& K. Viswanath (Eds.), Health behavior and health education: Theory research and practice (3rd ed., pp. 99-120). San Francisco, CA: Jossey-Bass.

Rothman, A., Bartels, R., Wlaschin, J., \& Salovey, P. (2006). The strategic use of gain- and loss-framed messages to promote healthy behavior: How theory can inform practice. Journal of Communication, 56, S202-S220.

Shafer, A., Cates, J. R., Diehl, S. J., \& Hartmann, M. (2011). Asking mom: Formative research for an HPV vaccine campaign targeting mothers of adolescent girls. Journal of Health Communication, 16(9), 988-1005.

Shen, L., \& Dillard, J. P. (2007). The influence of behavioral inhibition/approach systems and message framing on the processing of persuasive health messages. Communication Research, 34, 433-457.

Sherman, D., Mann, T., \& Updegraff, J. (2006). Approach/avoidance motivation, message framing, and health behavior: Understanding the congruency effect. Motivation and Emotion, 30(2), 164-168.

Sutton, S., \& Davidson, R. (1997). Prefrontal brain asymmetry: A biological substrate of the behavioral approach and inhibition systems. Psychological Science, 8(3), 204-210.

West, S. G., Aiken, L. S., \& Krull, J. L. (1996). Experimental personality designs: Analyzing categorical by continuous variable interactions. Journal of Personality, 48, 1-48.

Whittingham, J. R., Ruiter, R. A., Zimbile, F., \& Kok, G. (2008). Experimental pretesting of public health campaigns: A case study. Journal of Health Communication, 13, 216-229.

Zucker, N., Ferriter, C., Best, S., \& Brantley, A. (2005). Group parent training: A novel approach for the treatment of eating disorders. Eating Disorders, 13, 391-405.

How to cite this article: Shafer A, Patel S, Bulik C, Zucker N. Experimental pretesting of message framing to motivate caregiver self-care among parents of children with eating disorders. J Appl Behav Res.

2017;22:e12092. https://doi.org/10.1111/jabr.12092 